\title{
The Promise and Challenges of Personalized Medicine in Hematologic Malignancies
}

Keywords: Personalized medicine; Genomics; Diagnostics; Thera peutics; Healthcare

\begin{abstract}
Personalized medicine is a rapidly emerging medical model that proposes individually customized healthcare to potentially increase the quality of clinical care and reduce health care costs. The key to the success of personalized medicine is development of diagnostics and therapeutics based upon molecular, genetic, epigenetic, phamacogenomic and metabolic features of individual patients. Personalized medicine in oncology seeks to individualize cancer care and treatment decisions based upon features unique to each patient and each patient's tumor. Medic al decisions are then being tailored to the individual patient with the hope of providing improved quality-of-life and overall survival. Diagnostic testing is employed to select appropriate and optimal therapies based upon the context of each patient's genetic content or other molecular or cellular tests. The concept of personalized medicine hopes to not only enhance the patient quality of life and survival but also to lower overal healthcare costs through early-detection, prevention, accurate risk assessments and more efficient health care delivery systems. However, personalized approaches in oncology face major, complex scientific and economic problems. Here, we discuss certain scientific and economic challenges that face the future of personalized medic ine in the treatment of hematologic malignancies.
\end{abstract}

\section{Introduction}

Personalized medicine refers to the use of an individual patient's features to tailor treatments to each individual based on an analysis of one's molecular and genetic profile [1,2]. In general terms, personalized medicine (also known as precision medicine) is thought of as the tailoring of medical treatment to the individual characteristics of a patient during all stages of care, including prevention, diagnosis, treatment, and follow-up [3-5]. The term personalized medicine has been described as providing the right patient with the right drug at the right dose at the right time. The use of genetic information plays a major role in the design of personalized medicine and has been broadened to encompass all sorts of personalized treatment.

Personalized medicine may be considered an extension of current, traditional approaches to understanding and treating human cancers. Equipped with these tools that are more precise, physicians can now select a treatment protocol based on a patient's molecular profile that may not only minimize harmful side effects and ensure a more successful outcome, but can also help reduce adverse side effects and contain the high costs compared with the current trial-and-error approach. Personalized medicine also has the potential to change the way we think about, identify and manage health problems such as cancer.

\section{Scientific Challenges}

Cancer Genomics - The post-genomic era has fostered the

\section{Journal of}

Hematology \& Thrombosis

\section{James J. Driscoll ${ }^{1-3 *}$ and Troy Ludtka ${ }^{1}$}

${ }^{1}$ The Vontz Center for Molecular Studies, University of Cincinnati College of Medicine, Cincinnati, OH, USA

${ }^{2}$ Division of Hematology and Oncology, University of Cincinnati College of Medicine, Cincinnati, OH, USA

${ }^{3}$ Division of Hematology \& Oncology, University of Cincinnati Cancer Institute, Cincinnati, OH, USA

\section{*Address for Correspondence:}

James J. Driscoll, Division of Hematology \& Oncology, University of Cincinnati-College of Medicine, Cincinnati, $\mathrm{OH}, 45267-0508$, USA, Tel: (513)-558-2186; Fax: 513-558-670; E-mail: driscojs@uc.edu

\section{Submission: 18 July, 2015}

Accepted: 06 August, 2015

Published: 11 August, 2015

Copyright: (c) 2015 Driscoll JJ, et al. This is an open access article distributed under the Creative Commons Attribution License, which permits unrestricted use, distribution, and reproduction in any medium, provided the original work is properly cited.

Reviewed \& Approved by: Dr. Zartash Gul, Assistant Professor of Internal Medicine, Division of Hematology/Oncology and Bone Marrow Transplantation, University of Kentucky, USA

emergence of cutting-edge, systems-based approaches to elucidate the basic tenets of cancer biology and the complexities of chemo resistance that prevent patients from achieving sustained clinical responses. The identification of molecular determinants responsible for chemo resistance requires a shift from traditional biologic and genomic analyses to move the focus towards paradigm-shifting approaches and reveal genetic variations in critical pathways that control therapeutic response. Oncologists have long recognized that patients with the "same" cancer do not always respond to the "same" medicine. Each patient's cancer is unique, driven by distinct genetic disruptions and biological events.

Cancer researchers have realized a great deal about the genetic variety of types of cancer that appear the same in traditional pathology. Consequently, there has arisen an increased awareness of genetic diversity within a single tumor. These findings raise the possibility of finding actionable therapeutic targets and more effective drugs. While certain anti-cancer agents have not yielded significant benefit within unselected patient populations, individual cases may yet be successful for a proportion of cases with particular genetic profiles. Cancer Genomics (Oncogenomics) is the application of genomics and personalized medicine to cancer research and treatment. High-throughput sequencing methods are used to characterize genes associated with cancer to better understand disease pathology and improve drug development. Oncogenomics is one of the most promising branches of genomics, particularly because of its implications in drug therapy. Importantly, the key biological pathways that support uncontrolled tumor growth remain poorly characterized and the development and cost of anti-cancer medicines are very expensive. One of the most significant challenges 
in controlling cancer is the high degree of heterogeneity within the neoplastic cells of individual tumors. While tumor cell heterogeneity has been described at many levels and an understanding of the processes that create heterogeneity is emerging, the extent to which the heterogeneous subpopulations of tumor cells vary in their functional activities and whether interactions between subpopulation influence tumor initiation and progression is poorly understood. Major barriers to investigating these questions have been the lack of methodologies to maintain the heterogeneity of human tumors in culture and to propagate distinct clonal subpopulations from individual tumors.

Impact of intra tumoral heterogeneity on personalized medicines for hematologic cancers-pharmacogenomics will identify genetic and molecular variations in each patient's cancer cells to provide a greater understanding of the mechanisms of cancer and the molecular drivers of that person's unique cancer. Precision medicine is a transformative health care model that involves the selection of diagnostic tests with the potential to identify changes in each individual patient's cancer cells and to then identify personalized medicines.

The variability among cancer cells within a single neoplastic lesion has been known as intra tumor heterogeneity, which is increasingly evident in single-cell transcriptional profiling or single-cell sequencing [6-9]. The fundamental basis that underlies intra tumor heterogeneity is the molecular variability of the tissue microenvironment and neoplastic cells within a single tumor, in particular with epigenetic heterogeneity. Molecular heterogeneity within a single tumor poses a considerable challenge in cancer therapy, not only making prediction of tumor behavior very difficult, but also resulting in almost universal emergence of neoplastic clones resistant to a given therapy. Tumor progression has been regarded as an evolutionary process whereby molecular changes accumulate and clonal selection constantly takes place. Any molecular feature can be an advantage or disadvantage (even with very small effect) during the selection process, and this selection pressure may change at different tumor progression steps, depending on the tumor microenvironment. Data suggest that epigenetic alterations provide such diversity in intra tumor cell clonal populations perhaps due to a weaker selection pressure than driver mutations. A molecular feature that can give an advantage for cellular survival in the bloodstream may not be an advantage and may not be manifested until tumor cells invade deeply in tissue and blood vessel walls, and into the blood stream.

\section{Economic Challenges}

Current efforts to translate personalized healthcare into the clinic necessitates cooperation between the physician, the patient, the pharmaceutical industry, and the government and healthcare providers for effective decision-making. Such a paradigm hopes to increase the quality of clinical care and treatments and ultimately to save costs. This highlights the imperative for a closer collaboration between bench scientists, clinical investigators and pharmaceutical companies to prioritize the mechanism of action of a potential drug candidate when deciding which group of patients should be treated with the investigational agent on experimental protocols. Approaches to intellectual property rights, reimbursement policies, patient privacy and confidentiality also will be affected by a personalized approach to healthcare. Furthermore, effective implementation of personalized medicine will require resources to analyze acquired diagnostic data, storage of electronic medical records and patient-centered outcomes research to improve the quality and relevance of evidence available to help patients, caregivers, clinicians, employers, insurers and policy makers make informed health decisions. Next-generation sequencing and bioinformatic analyses will require extensive costs for data processing and storage.

Having an individual's genomic information can be significant in the process of developing drugs as they await approval from the FDA for public use. A detailed account of an individual's genetic make-up can be a major asset in deciding if a patient can be chosen for inclusion or exclusion in the final stages of a clinical trial. Being able to identify patients who will benefit most from a clinical trial will increase the safety of patients from adverse outcomes caused by the product in testing, and will allow smaller and faster trials that lead to lower overall costs. Drugs that are deemed ineffective for the greater population of cancer patients can gain FDA approval by through correlation with personalized genomes to qualify the effectiveness and need for that specific drug or therapy even though it may only be needed by a small percentage of the population.

\section{Conclusions}

To standardize personalized medicine a widespread, accepted procedure in clinical practice, physicians should closely collaborate to reshape the objectives to provide a basis for better and more efficient drug development and patient treatment. Healthcare system reforms that promote more flexible and value-based reimbursement for innovative diagnostics and therapeutics are needed to generate economic incentives for personalized medicine development. Despite our knowledge of the existence of tumor cell heterogeneity, it is not understood whether the heterogeneous subpopulations of tumor cells merely co-exist, or alternatively whether they communicate with each other, complementing one another's phenotypes and generating biological outcomes that individual populations are incapable of producing on their own. The lack of understanding of such functional interactions between tumor populations has been due in large part to the inability to maintain the heterogeneity of human tumors in culture and to propagate distinct clonal subpopulations from individual tumors. The promise is that the focus of medicine will be shifted from disease to wellness and that thousands of data points for each individual patient will be taken into account and defines with exquisite specificity the nature of their wellness and any transitions into disease. Central to this view is the idea that the molecular, cellular, genomic and phenotypic data of eventually thousands of patients will be available for complex systems analyses, i.e. integration, normalizing, query, mining, analysis, storage and protection of data.

A vision for precision medicine to treat patients diagnosed with hematologic malignancies calls for the deployment of molecularly targeted therapeutics in genetically defined patient populations. A first step in that process involves an accurate description of the genetic landscape of human blood cancers. While virtually all human tumors are derived from a single cell-of-origin, neoplastic cells within a tumor evolve over time due to genetic and epigenetic alterations, lineage diversification, and influences from stromal cells. Understanding these processes that yield considerable heterogeneity within populations of neoplastic cells from individual tumors will 
Citation: Driscoll JJ, Ludtka T. The Promise and Challenges of Personalized Medicine in Hematologic Malignancies. J Hematol Thromb 2015;1(2): 3.

ISSN: 2380-6842

eventually lead to personalized medicines that improve patient survival.

\section{References}

1. Guttmacher AE, Collins FS (2005) Realizing the promise of genomics in biomedical research. JAMA 294: 1399-1402.

2. Collins FS, Varmus $\mathrm{H}$ (2015) A new initiative on precision medicine. N Engl J Med 372: 793-795

3. Lu YF, Goldstein DB, Angrist M, Cavalleri G (2014) Personalized medicine and human genetic diversity. Cold Spring Harbor Perspect Med 4: a008581.

4. Dudley JT, Karczewski KJ (2014) Exploring personal genomics. Oxford: Oxford University Press, United Kingdom.
5. Martinez de Lecea MG, Michael R (2012) Translational genomics in personalized medicine-scientific challenges en route to clinical practice. HUGO J 6: 2.

6. Fidler IJ (1978) Tumor heterogeneity and the biology of cancer invasion and metastasis. Cancer Res 38: 2651-2660.

7. Berman HK, Gauthier ML, TIsty TD (2010) Premalignant breast neoplasia: a paradigm of interlesional and intralesional molecular heterogeneity and its biological and clinical ramifications. Cancer Prev Res (Phila) 3: 579-587.

8. Merlo LM, Pepper JW, Reid BJ, Maley CC (2006) Cancer as an evolutionary and ecological process. Nat Rev Cancer 6: 924-935.

9. Campbell LL, Polyak K (2007) Breast tumor heterogeneity: cancer stem cells or clonal evolution? Cell Cycle 6: 2332-2338.

\section{Acknowledgements}

The authors acknowledge the support of the University of Cincinnati Division of Hematology and Oncology for funding provided through the Hematology and Oncology Translational Studies Award. 